\title{
Asset Prices and Changes in Risk within a Bivariate Model
}

\author{
Octave Jokung $^{1} \cdot$ Sovan Mitra ${ }^{2}$ (I)
}

Published online: 9 November 2018

(c) The Author(s) 2018

\begin{abstract}
This paper analyzes the effect on the price of the risky asset for both global and marginal changes, in dependent, independent, exogenous and endogenous risks. We find that global changes induce riskier behavior and decrease market prices when the utility function exhibits generalized relative risk aversions less than their benchmark values. Marginal changes in the endogenous risk decrease the market prices when all the coefficients of generalized relative risk aversion to the endogenous risk are less than their orders. Marginal changes in the exogenous risk decrease the price of the risky asset, when all the coefficients of generalized risk aversion to the exogenous risk are less than one. Positive dependence induces a decrease in the market price whereas negative dependence causes an increase in the same price. Furthermore, increasing the correlation between the two risks leads to the fall in the price of the risky asset, when the utility function of the representative investor exhibits pair-wise risk aversion. We recover the results concerning the univariate framework with additive background risk.
\end{abstract}

Keywords Monotonicity · Background risk - Increase in risk · Pair-wise risk aversion · Risk aversion · Correlation

\section{Introduction}

Several papers such as Dionne et al. (1995), Eeckhoudt et al. (2016), Gollier (1995), Gollier and Kimball (2018), Iwaki and Osaki (2017), Choi et al. (2001), Gollier and Schlesinger (2002) and Ohnishi and Osaki (2007) amongst others have examined the effect of a change in the return of a risky asset on its price. They give the conditions on preferences of the decision-maker or on the distribution of the risky asset to guarantee a decrease in the price, after an increase in the risk. Except for Osaki

\footnotetext{
Sovan Mitra

sovan.mitra@liverpool.ac.uk

1 IAE School of Management, University of Valenciennes, Valenciennes, France

2 Department of Mathematics, University of Liverpool, Liverpool, UK
} 
(2005), who examines the effect of a dependent background risk on the asset price, they only consider one source of risk. Osaki (2005), however, restricts the analysis to background risks with functional forms. In this paper we extend this approach to stochastic dependent background risk. In fact, we consider a bivariate risk in which the first risk corresponds to the return on the risky asset, and the second risk represents the background risk or in fact any exogenous risk.

In general, decisions under risk are multidimensional because the decision-maker faces several risks and his preferences depend on several attributes. However, the attitude towards risk in the bivariate framework is not obtained in a straightforward manner from that of the univariate framework. This is because the interaction between the attributes of the utility function matters. For instance, Richard (1975) defines multivariate risk aversion with preferences over two lotteries and shows that the key factor in determining the choice between the lotteries is the way in which the attributes are coupled. The lottery with two "bad" attributes or two "good" attributes is dominated by the lottery with one "bad" and one "good" attribute. Therefore the decision-maker prefers getting some of the "good" and some of the "bad", compared to taking a chance on getting all of the "good" or all the "bad". Scarsini (1985) calls this interaction concept 'pair-wise risk aversion', and Epstein and Tanny (1980) call it 'correlation aversion'.

In this paper, we analyze the monotonicity of the asset price with regards to the bivariate risk. Specifically, we find the conditions on preferences to guarantee a decline in the price of the risky asset after deterioration in the bivariate risk. We find that the deterioration is said to be marginal when we increase one of the two risks via $k$-th degree stochastic dominance. The deterioration will be global if we increase both risks. We can illustrate our findings with an example from the case of health and wealth: Palombo (1999) shows that uncertain health expenses generate precautionary savings. Also, Pang and Warshawsky (2010) demonstrates that uninsured health expenses lower non-health consumption, at all ages, and that the wealth available for consumption after deduction of health expenses becomes more volatile in presence of additional background risk. The uncertainty in health expenses imply precautionary savings and a shift from risky assets to risk-free assets. Other examples include Edwards (2008) who presents a theoretical model in which portfolio shares are based on health risk, Fan and Zhao (2009) show that adverse health shocks motivate a safer portfolio choice. In other words, health shocks shift investment from risky assets toward less risky assets. Meer et al. (2003) analyze the impact of wealth on health status and show that wealth-health connection is not driven by short run changes in wealth. However, changes in wealth have a strong correlation with changes in health. All these papers highlight the relationship between health status and portfolio selection generally with empirical studies.

Our paper contributes to this literature in several ways. First, we show that in the case of increases in both endogenous and exogenous risks, the price of the risky asset decreases. Second, we generalize several results concerning the effect of the exogenous risk and the endogenous risk on the market price. Third, we recover the results concerning the univariate framework with additive background risks. Fourth, we explain the effect of the correlation and that of the change in the correlation on the price of the risky asset. 
The paper proceeds as follows: Sect. 2 presents the model with the two sources of risks, in Sect. 3 we present the definitions of stochastic dominance. In Sect. 4, we give conditions on preferences to ensure that a global increase in risk (change in the bivariate risk) induces riskier behavior and leads to a decrease in the market price. We also consider the special case of marginal changes: change in the endogenous risk or change in the exogenous risk. We give conditions under which the market price declines after the alteration of the endogenous risk as a result of the $N$ th-order stochastic dominance. We present the conditions on preferences to ensure that an increase in the exogenous risk via the $M$ th-order stochastic dominance induces a decrease in the price of the risky asset. In the next section, we analyze the effect of the correlation of the two sources of risks and we give conditions under which an increase in the correlation causes a decrease in the market price. Finally, we end with a conclusion.

\section{Introduction to the Risk Model}

We consider a static version of a Lucas (1978) pure-exchange economy with two periods and homogenous investors. We assume that the representative investor trades one risk-free asset and one risky asset. He is endowed with $w$ units of the risk-free asset and one unit of the risky asset. We assume, as usual, that the risk-free asset is the numeraire and the gross risk-free rate is normalized to one. The final value of the risky asset is given by a random variable $X$ taking its values in the interval $[\underline{x}, \overline{\mathrm{x}}]$ with the distribution function $F(x)$. We call the risky asset, the endogenous risk. We now introduce the exogenous risk $Y$ with cumulative distribution function $G(y)$ in the interval $[\underline{\mathrm{y}}, \overline{\mathrm{y}}]$ and we let $H(x, y)$ be the cumulative joint distribution of $(X, Y)$.

The representative investor chooses the optimal percentage of his wealth, $\alpha$, invested in the risky asset, such that the expected utility of his final wealth is maximized. We can express this as:

$$
\max _{\alpha} E u[\alpha X+(1-\alpha) P, Y]=\int_{\underline{\mathrm{y}}}^{\overline{\mathrm{y}}} \int_{\underline{\underline{x}}}^{\overline{\mathrm{x}}} u(\alpha x+(1-\alpha) P, y) d H(x, y),
$$

where $P$ represents the price of the risky asset.

The utility function $u$ is at least twice differentiable with

$$
\begin{aligned}
& u^{(1,0)}(x, y)=\frac{\partial u(x, y)}{\partial x} \geq 0, \\
& u^{(0,1)}(x, y)=\frac{\partial u(x, y)}{\partial y} \geq 0,
\end{aligned}
$$


and

$$
u^{(2,0)}(x, y)=\frac{\partial^{2} u(x, y)}{\partial x^{2}} \leq 0
$$

Therefore the first-order condition of this problem is:

$$
\int_{\underline{\mathrm{y}}}^{\overline{\mathrm{y}}} \int_{\underline{\mathrm{x}}}^{\overline{\mathrm{x}}}(x-P) u^{(1,0)}(\alpha x+(1-\alpha) P, y) d H(x, y)=0
$$

Or equivalently:

$$
\int_{\underline{\mathrm{y}}}^{\overline{\mathrm{y}}} \int_{\underline{\mathrm{x}}}^{\overline{\mathrm{x}}} x u^{(1,0)}(\alpha x+(1-\alpha) P, y) d H(x, y)=\int_{\underline{\mathrm{y}}}^{\overline{\mathrm{y}}} \int_{\underline{\underline{\mathrm{x}}}}^{\overline{\mathrm{x}}} P u^{(1,0)}(\alpha x+(1-\alpha) P, y) d H(x, y)
$$

Therefore, the price of the risky asset is given by:

$$
P=\frac{\int_{\underline{y}}^{\bar{y}} \int_{\underline{x}}^{\bar{x}} x u^{(1,0)}(\alpha x+(1-\alpha) P, y) d H(x, y)}{\int_{\underline{y}}^{\bar{y}} \int_{\underline{x}}^{\bar{x}} u^{(1,0)}(\alpha x+(1-\alpha) P, y) d H(x, y)}
$$

The second-order condition is fulfilled as a result of the concavity of the utility function with respect to its first attribute. To determine the price of the risky asset, we use the market-clearing condition: $\alpha=1$. The equilibrium price is then given by (see Gollier and Schlesinger 2002):

$$
P=\frac{\int_{\underline{\mathrm{y}}}^{\overline{\mathrm{y}}} \int_{\underline{\mathrm{x}}}^{\overline{\mathrm{x}}} x u^{(1,0)}(x, y) d H(x, y)}{\int_{\underline{\mathrm{y}}}^{\overline{\mathrm{y}}} \int_{\underline{\mathrm{x}}}^{\overline{\mathrm{x}}} u^{(1,0)}(x, y) d H(x, y)}
$$

Now the equation is equivalent to $E X u^{(1,0)}(X, Y)$ divided by $E X u^{(1,0)}(X, Y)$, hence we can write

$$
P=\frac{E X u^{(1,0)}(X, Y)}{E u^{(1,0)}(X, Y)}
$$

Hence the equilibrium price is given by the ratio of two expectations.

\section{Stochastic Dominance Preliminaries}

In this section we introduce stochastic dominance preliminaries. Stochastic dominance is a method of partial ordering of random variables, in a set. It is particularly used 
in decision theory when applied to ranking one investment as superior to another investment, for a wide range of decision makers. Stochastic dominance allows us to determine if a particular random variable is considered riskier than another random variable. Such properties cannot always be derived for a broad class of decision makers using other methods of decision analysis.

In the univariate case, consider two random variables $X$ and $Y$ with respective cumulative distribution functions $F$ and $G$, with bounded supports contained within the interval $[a, b]$. Let

$$
\begin{aligned}
& F_{1}(x)=F(\mathrm{x}) \text { and } G_{1}(x)=G(\mathrm{x}) \\
& F_{n}(x)=\int_{a}^{x} F_{n-1}(z) d z \text { and } G_{n}(x)=\int_{a}^{x} G_{n-1}(z) d z, \quad \forall n \geq 2 .
\end{aligned}
$$

Definition 1 The distribution $F$ or equivalently the random variable $X$, dominates the distribution $G$ or equivalently the random variable $Y$, in the sense of the Nth-order stochastic dominance if and only if:

$$
F_{N}(x) \leq G_{N}(x), \quad \forall x \in[a, b],
$$

and

$$
F_{n}(b) \leq G_{n}(b), \quad \forall n=1, \ldots, N-1 .
$$

We write $F N S D G$ to denote that $F$ dominates $G$ with respect to the $N$ th-order stochastic dominance. $N=1, N=2$ and $N=3$ correspond to the first-order stochastic dominance (FSD), second-order stochastic dominance (SSD) and third-order stochastic dominance (TSD). First order stochastic dominance means that a random variable (or investment) $Y$ dominates random variables $X$, if and only if every expected utility maximiser with an increasing utility function prefers $Y$ over $X$. For second order stochastic dominance, $Y$ has dominance over $X$ if $Y$ is more predictable (or has less risk) than $X$ but has at least the same expectation.

In all what follows $v^{(n)}(w)$ is the $n$th derivative of the univariate utility function $v$. We have the well-known following result.

Theorem 1 The following are equivalent

(i) $F N S D G(X N S D Y)$.

(ii) $\int_{a}^{b} v(z) d F(z) \geq \int_{a}^{b} v(z) d G(z), \quad \forall v$, such that $(-1)^{n+1} v^{(n)}(w) \geq 0, \quad \forall n=1, \ldots, N$.

Consider two bivariate random variables $\left(X_{1}, Y_{1}\right)$ and $\left(X_{2}, Y_{2}\right)$ with respective cumulative joint distributions $H^{(1)}(x, y)$ and $H^{(2)}(x, y)$. Let us define like Denuit et al. (1999), the class of $(N, M)$-increasing concave functions, denoted by $\mathcal{U}_{N, M-i c v}$ as follows. 
Definition $2 u \in \mathcal{U}_{N, M-i c v}$ if and only if

$$
(-1)^{n+m+1} u^{(n, m)}(x, y) \geq 0, \quad \forall n=0, \ldots, N, \quad \forall m=0, \ldots, M ; n+m \geq 1
$$

with $u^{(n, m)}(x, y)=\frac{\partial^{n+m} u(x, y)}{\partial x^{n} \partial y^{m}}$ the $(n, m)$ th partial derivative of the bivariate utility function $\mathrm{u}$.

The common preferences of all the decision-makers with $(N, M)$-increasing concave utility functions generate the $(N, M)$-increasing concave dominance rule, termed the $(N, M)$-increasing concave order. Denuit et al. (1999) give the following definition.

Definition $3\left(X_{1}, Y_{1}\right)$ dominates $\left(X_{2}, Y_{2}\right)$ in the $(N, M)$-increasing concave order, denoted by $\left(X_{2}, Y_{2}\right) \preccurlyeq N, M-i c v\left(X_{1}, Y_{1}\right)$, when for all functions $u$ in $\mathcal{U}_{N, M-i c v}$, we have $E u\left(X_{2}, Y_{2}\right) \leq E u\left(X_{1}, Y_{1}\right)$.

\section{Risk Model and Changes in the Bivariate Risk}

In this section we expound our key theoretical result in terms of the risk model, which gives us a framework for analyzing changes in risk. Consequently, in the proceeding subsections, we apply our framework to understanding changes in endogenous risk and exogenous risk.

\subsection{Risk Theorem}

Let $P_{1}^{*}$ and $P_{2}^{*}$ be the optimal prices with $\left(X_{1}, Y_{1}\right)$ and $\left(X_{2}, Y_{2}\right)$, respectively. If we know that $\left(X_{2}, Y_{2}\right) \preccurlyeq N, M-i c v\left(X_{1}, Y_{1}\right)$, then we want to give conditions under which deterioration in the bivariate risk induces a decrease in the price of the risky asset. We now have the following result.

Theorem 2 If $u \in \mathcal{U}_{N+1, M-i c v}$ and $\forall n=1, \ldots, N$

$$
-x \frac{u^{(n+1, m)}}{u^{(n, m)}}(x, y) \leq n, \quad \forall x, y, \quad \forall m=0, \ldots, M
$$

then

$$
\left(X_{2}, Y_{2}\right) \preccurlyeq_{N, M-i c v}\left(X_{1}, Y_{1}\right) \Rightarrow P_{2}^{*} \leq P_{1}^{*} .
$$

Proof We have

$$
\begin{aligned}
& \int_{\underline{\mathrm{y}}}^{\overline{\mathrm{y}}} \int_{\underline{\mathrm{x}}}^{\overline{\mathrm{x}}} u^{(1,0)}(x, y) d H^{(1)}(x, y) \leq \int_{\underline{\mathrm{y}}}^{\overline{\mathrm{y}}} \int_{\underline{\mathrm{x}}}^{\overline{\mathrm{x}}} u^{(1,0)}(x, y) d H^{(2)}(x, y) \\
& \quad \Leftrightarrow-E u^{(1,0)}\left(X_{1}, Y_{1}\right) \geq-E u^{(1,0)}\left(X_{2}, Y_{2}\right)
\end{aligned}
$$


which is true if $-u^{(1,0)} \in \mathcal{U}_{N, M-i c v}$. That is,

$$
(-1)^{n+m+2} u^{(n+1, m)}(x, y) \geq 0, \quad \forall n=0, \ldots, N, \quad \forall m=0, \ldots, M ; n+m \geq 1
$$

This condition holds if $\in \mathcal{U}_{N+1, M-i c v}$.

Therefore, $P_{2}^{*} \leq P_{1}^{*}$ if

$$
\begin{aligned}
& \int_{\underline{y}}^{\overline{\mathrm{y}}} \int_{\underline{\mathrm{x}}}^{\overline{\mathrm{x}}} x u^{(1,0)}(x, y) d H^{(1)}(x, y) \geq \int_{\underline{\mathrm{y}}}^{\overline{\mathrm{y}}} \int_{\underline{\mathrm{x}}}^{\overline{\mathrm{x}}} x u^{(1,0)}(x, y) d H^{(2)}(x, y) \\
& \Leftrightarrow E X_{1} u^{(1,0)}\left(X_{1}, Y_{1}\right) \geq E X_{2} u^{(1,0)}\left(X_{2}, Y_{2}\right)
\end{aligned}
$$

which is true when $\Phi(x, y)=x u^{(1,0)}(x, y) \in \mathcal{U}_{N, M-i c v}$. That is,

$$
\begin{gathered}
(-1)^{n+m+1} u^{(n, m)}(x, y)\left[n+x \frac{u^{(n+1, m)}}{u^{(n, m)}}(x, y)\right] \geq 0 \\
\forall n=0, \ldots, N, \quad \forall m=0, \ldots, M ; n+m \geq 1 .
\end{gathered}
$$

Equivalently,

$$
\left[n+x \frac{u^{(n+1, m)}}{u^{(n, m)}}(x, y)\right] \geq 0, \quad \forall n=1, \ldots, N, \quad \forall m=0, \ldots, M ; n+m \geq 1
$$

due to the fact that $u \in \mathcal{U}_{N, M-i c v}$.

Finally, the condition to be fulfilled is the following:

$$
-x \frac{u^{(n+1, m)}}{u^{(n, m)}}(x, y) \leq n, \quad \forall n=1, \ldots, N, \quad \forall m=0, \ldots, M .
$$

Let us consider the generalized coefficient of relative risk aversion of order $(n, m)$ with respect to the first attribute of the utility function and defined as follows:

$$
-x \frac{u^{(n+1, m)}}{u^{(n, m)}}(x, y)
$$

The condition to guarantee a decrease in the price of the risky asset after a deterioration of the bivariate risk is that this coefficient must be less than its benchmark value given by $n$. In the univariate framework, Eeckhoudt et al. (2009) show that the respective benchmark values of relative risk aversion and relative prudence are one and two. In fact, the benchmark value for $-w \frac{v^{(n+1)}(w)}{v^{(n)}(w)}$ is $n$. Therefore, the condition says that for all values of $m$ up to $M$, the coefficient of relative risk aversion of $u^{(0, m)}$ with respect to the first attribute at any order $n$ up to $N$, is less than its order given by $n$. 


\subsection{Change in the Endogenous Risk}

Our framework allows us to analyze the change in the endogenous risk in the presence of the exogenous risk. Let us consider the following problems for $i=1,2$ :

$$
\max _{\alpha_{i}} E u\left(\alpha_{i} X_{i}+\left(1-\alpha_{i}\right) P_{i}, Y_{1}\right)
$$

The change in the endogenous risk $X$ from $X_{1}$ to $X_{2}$ via $N$ th-degree stochastic dominance is common in the literature. We want to clarify the conditions under which a deterioration of the endogenous risk causes a decrease in the market price, in the presence of the exogenous risk. The change in the endogenous risk is equivalent to the comparison of the optimal decision when the representative investor faces $\left(X_{1}, Y_{1}\right)$ denoted by $P_{1}$ *, with that when he faces $\left(X_{2}, Y_{1}\right)$ denoted by $P_{2}{ }^{*}$, and knowing that $\left(X_{2}, Y_{1}\right) \preccurlyeq_{N, 1-i c v}\left(X_{1}, Y_{1}\right)$. We now have the following result.

Proposition 1 If $u \in \mathcal{U}_{N+1,1-i c v}$ with

$$
-x \frac{u^{(n+1,1)}}{u^{(n, 1)}}(x, y) \leq n, \quad \forall n=1, \ldots, N,
$$

then

$$
\left(X_{2}, Y_{1}\right) \preccurlyeq N, 1-i c v\left(X_{1}, Y_{1}\right) \Rightarrow P_{2}^{*} \leq P_{1}^{*}
$$

Proof The proof is direct by applying Theorem 2 .

The market price decreases after deterioration in the endogenous risk via $N$ thdegree stochastic dominance when: all the coefficients of relative risk aversion of the marginal utility with respect to the attribute not concerned by the risky asset are less than their benchmark values up to the degree corresponding to the stochastic dominance in question.

To recover the result concerning additive risks, we apply Proposition 1 to the bivariate utility function $u(z, y)=v(z+y)$ and we get:

$$
\text { if }(-1)^{n-1} v^{n}(w) \geq 0, \quad \forall n=1, \ldots, N
$$

and

$$
-x \frac{v^{(n+1)}}{v^{(n)}}(x+y) \leq n, \quad \forall x, y, \quad \forall n=1, \ldots, N,
$$

then

$$
\left(X_{2}, Y_{1}\right) \preccurlyeq_{N, 1_{-i c v}}\left(X_{1}, Y_{1}\right) \Rightarrow P_{2}^{*} \leq P_{1}^{*}
$$

Equivalently, knowing that $X_{1}$ dominates $X_{2}$ via $N$ th-degree stochastic dominance, the representative investor reduces the holding in risky asset if $(-1)^{n-1} v^{n}(w) \geq$ $0, \forall n=1, \ldots, N$, and $-x \frac{v^{(n+1)}}{v^{(n)}}(x+y) \leq n, \forall x, y, \forall n=1, \ldots, N$. We also have the following result. 
Corollary 1 The deterioration in the endogenous risk via the Nth-degree stochastic dominance reduces the market price if the successive derivatives of the utility function alternate in sign, the first being positive and $-x \frac{v^{(n+1)}}{v^{(n)}}(x+y) \leq n, \forall x, y, \forall n=$ $1, \ldots, N$.

The proposition remains true if we consider relative risk aversion instead of partial relative risk aversion.

Consider the degenerate exogenous risk $E\left(Y_{1}\right)$ and compare the situation with $\left(X_{1}, E\left(Y_{1}\right)\right)$ with that with $\left(X_{2}, E\left(Y_{1}\right)\right)$ knowing that $X_{1}$ dominates $X_{2}$ via first-order stochastic dominance. The conditions become $u^{\prime} \geq 0, u^{\prime \prime} \leq 0$ and $-x \frac{v^{\prime \prime}}{v^{\prime}}(x+y) \leq 1$. We recover the results from Fishburn and Porter (1976) and Cheng et al. (1987) with first-order stochastic dominance. If we consider second-order stochastic dominance, we end up with that from Ohnishi and Osaki (2007). In the former the coefficient of relative risk aversion must be less than one whereas in the latter the coefficient of relative prudence must, in addition, be less than two.

\subsection{Change in the Exogenous Risk}

The change in the exogenous risk is equivalent to the comparison of the price of the risky asset when the representative investor faces $\left(X_{1}, Y_{1}\right)$ denoted by $P_{1}$ *, with that when he faces $\left(X_{1}, Y_{2}\right)$ denoted by $P_{2}^{*}$, and knowing that $\left(X_{1}, Y_{1}\right) \preccurlyeq 1, M-i c v\left(X_{1}, Y_{2}\right)$. We now have the following result.

Proposition 2 If $u \in \mathcal{U}_{2, M-i c v}$ with

$$
-x \frac{u^{(2, m)}}{u^{(1, m)}}(x, y) \leq 1, \forall m=0, \ldots, M,
$$

then

$$
\left(X_{1}, Y_{2}\right) \preccurlyeq 1, M-i c v\left(X_{1}, Y_{1}\right) \Rightarrow P_{2}^{*} \leq P_{1}^{*}
$$

Proof The proof is direct by applying Theorem 2 .

The former proposition in the event of independence between the endogenous and the exogenous risk becomes the effect of an $M$ th-degree stochastic dominance increases in the exogenous risk on the market price. For example, deterioration of the exogenous risk via first-order stochastic dominance leads to a decrease in the market price if the utility function belongs to $\mathcal{U}_{2,1_{-i c v}}$ and relative cross-prudence with respect to the first attribute of the utility function (that having to do with the risky asset) is less than one. With SSD, the additional condition is a relative cross-temperance with respect to the attribute of the utility that having to do with the risky asset less than one.

To obtain the result concerning the univariate framework with an additive background risk, let us again consider the following utility function which accounts for additive risks:

$$
u(x, y)=v(x+y) .
$$


The conditions in Proposition 2 become:

$$
u \in \mathcal{U}_{2, M-i c v} \Leftrightarrow(-1)^{m} v^{(m+1)}(x+y) \geq 0, \quad \forall m=0, \ldots, M
$$

and

$$
-x \frac{u^{(2, m)}}{u^{(1, m)}}(x, y) \leq 1, \quad \forall m=0, \ldots, M \stackrel{\Leftrightarrow}{:}-x \frac{v^{(m+2)}}{v^{(m+1) ! \prime \prime}}(x+y) \leq 1, \quad \forall m=0, \ldots, M
$$

Therefore, we recover the result of the effect of the change in the additive background with respect to $M$ th-degree stochastic dominance in the univariate framework.

Corollary 2 The deterioration of the additive background risk via Mth-degree stochastic dominance reduces the market price if the successive derivatives of the utility function alternate in sign, the first being positive, and all the coefficients of relative risk aversion of order less than $M+1$ are less than one.

\section{Risk and the Effect of Dependence: Changes in the Correlation}

Now, we want to know whether the dependence between the exogenous risk and the endogenous risk affects the price of the risky asset. To do so, we consider the case of Bernoulli-distributed variables and the concept of increasing correlation. We also present the result concerning the additive correlated background risk in the univariate framework.

\subsection{Bernoulli-Distributed Variables and the Effect of Dependence}

Assume that $X$ and $Y$ take two values $x_{1}$ and $x_{2}$ for $X$, with $x_{1} \leq x_{2}$, and $y_{1}$ and $y_{2}$ for $Y$, with $y_{1} \leq y_{2}$. The joint distribution of $(X, Y)$ is given by:

$$
\begin{aligned}
& P\left(X=x_{1}, Y=y_{1}\right)=k p q, \\
& P\left(X=x_{1}, Y=y_{2}\right)=p(1-k q), \\
& P\left(X=x_{2}, Y=y_{1}\right)=q(1-k p), \\
& P\left(X=x_{2}, Y=y_{2}\right)=1-p-q+k p q .
\end{aligned}
$$

The marginal distribution of $X$ is given by: $P\left(X=x_{1}\right)=p$ and $P\left(X=x_{2}\right)=1-$ p. The marginal distribution of $Y$ is given by: $P\left(Y=y_{1}\right)=q$ and $P\left(Y=y_{2}\right)=1-q$. There are three cases depending on the value of the dependence parameter $k$ :

$k>1 \quad X$ and $Y$ are positively correlated (positive dependence);

$k=1 \quad X$ and $Y$ are independent;

$k<1 \quad X$ and $Y$ are negatively correlated (negative dependence).

Let us define the bivariate risk $\left(X^{\perp}, Y^{\perp}\right)$ such that:

- $X^{\perp}$ and $X$ have the same distribution, 
$-Y^{\perp}$ and $Y$ have the same distribution and

$-X^{\perp}$ and $Y^{\perp}$ are independent.

Let $P_{\perp} *$ be the market price in the event of independence. We have the following proposition.

Theorem 3 If $u \in \mathcal{U}_{2,1-i c v}$ with

$$
-x \frac{u^{(2,0)}}{u^{(1,0)}}(x, y) \leq 1, \quad-x \frac{u^{(2,1)}}{u^{(1,1)}}(x, y) \leq 1, \quad \forall x, y
$$

(i) If $X$ and $Y$ are positively correlated, then $P^{*} \leq P_{\perp} *$.

(ii) If $X$ and $Y$ are negatively correlated, then $P^{*} \geq P_{\perp} *$.

Proof

$$
\begin{aligned}
E\left[u^{(1,0)}(X, Y)\right]= & E\left[u^{(1,0)}\left(X^{\perp}, Y^{\perp}\right)\right]+(k-1) p q\left[u^{(1,0)}\left(x_{1}, y_{1}\right)\right. \\
& \left.-u^{(1,0)}\left(x_{1}, y_{2}\right)-u^{(1,0)}\left(x_{2}, y_{1}\right)+u^{(1,0)}\left(x_{2}, y_{2}\right)\right] \\
E\left[X u^{(1,0)}(X, Y)\right]= & E\left[X^{\perp} u^{(1,0)}\left(X^{\perp}, Y^{\perp}\right)\right]+(k-1) p q\left[x_{1} u^{(1,0)}\left(x_{1}, y_{1}\right)\right. \\
& \left.-x_{1} u^{(1,0)}\left(x_{1}, y_{2}\right)-x_{2} u^{(1,0)}\left(x_{2}, y_{1}\right)+x_{2} u^{(1,0)}\left(x_{2}, y_{2}\right)\right]
\end{aligned}
$$

We have:

$$
\begin{aligned}
E\left[u^{(1,0)}(X, Y)\right] & =E\left[u^{(1,0)}\left(X^{\perp}, Y^{\perp}\right)\right]+(k-1) p q \Delta, \\
E\left[X u^{(1,0)}(X, Y)\right] & =E\left[X^{\perp} u^{(1,0)}\left(X^{\perp}, Y^{\perp}\right)\right]+(k-1) p q \Pi .
\end{aligned}
$$

$\Delta$ is non-positive if and only if $u^{(1,0)}$ exhibits pair-wise risk aversion, as defined by Richard (1975) and Scarsini (1985). Or, equivalently if and only if $u^{(2,1)} \leq 0$. $\Pi$ is non-positive if and only if $x u^{(1,0)}(x, y)$ is sub-modular, that is, $x u^{(1,0)}(x, y)$ exhibits pair-wise risk aversion. Assume that $u^{(1,1)} \leq 0$ :

The function $x u^{(1,0)}(x, y)$ is sub-modular if and only if $u^{(1,1)}(x, y)+x u^{(2,1)}(x, y) \leq 0$.

Equivalently, $-x \frac{u^{(2,1)}(x, y)}{u^{(1,1)}(x, y)} \leq 1$.

First case: $X$ and $Y$ are positively correlated $(k>1)$.

Assume that $-x \frac{u^{(2,1)}(x, y)}{u^{(1,1)}(x, y)} \leq 1$ and $u^{(2,1)} \geq 0$. Then $\Delta \geq 0$ and $\Pi \leq 0$. Thus:

$$
\begin{aligned}
E\left[u^{(1,0)}(X, Y)\right] & \geq E\left[u^{(1,0)}\left(X^{\perp}, Y^{\perp}\right)\right] \\
E\left[X u^{(1,0)}(X, Y)\right] & \leq E\left[X^{\perp} u^{(1,0)}\left(X^{\perp}, Y^{\perp}\right)\right]
\end{aligned}
$$

Therefore,

$$
\frac{E\left[X u^{(1,0)}(X, Y)\right]}{E\left[u^{(1,0)}(X, Y)\right]} \leq \frac{E\left[X^{\perp} u^{(1,0)}\left(X^{\perp}, Y^{\perp}\right)\right]}{E\left[u^{(1,0)}\left(X^{\perp}, Y^{\perp}\right)\right]}
$$


meaning that $P^{*} \leq P_{\perp} *$.

Second case: $X$ and $Y$ are negatively correlated $(k<1)$.

Assume that $-x \frac{u^{(2,1)}(x, y)}{u^{(1,1)}(x, y)} \leq 1$ and $u^{(2,1)} \geq 0$. Then, $\Delta \leq 0$ and $\Pi \geq 0$. Thus:

$$
\begin{aligned}
E\left[u^{(1,0)}(X, Y)\right] & \leq E\left[u^{(1,0)}\left(X^{\perp}, Y^{\perp}\right)\right], \\
E\left[X u^{(1,0)}(X, Y)\right] & \geq E\left[X^{\perp} u^{(1,0)}\left(X^{\perp}, Y^{\perp}\right)\right] .
\end{aligned}
$$

Therefore

$$
\frac{E\left[X u^{(1,0)}(X, Y)\right]}{E\left[u^{(1,0)}(X, Y)\right]} \geq \frac{E\left[X^{\perp} u^{(1,0)}\left(X^{\perp}, Y^{\perp}\right)\right]}{E\left[u^{(1,0)}\left(X^{\perp}, Y^{\perp}\right)\right]}
$$

meaning that $P^{*} \geq P_{\perp} *$.

If we consider the utility function which accounts for additive risks $(u(x, y)=v(x$ $+y)$ ), the conditions in Theorem 3 become:

$$
\begin{aligned}
v^{\prime}(x+y) & \geq 0, \\
v^{\prime \prime}(x+y) & \leq 0, \\
v^{\prime \prime \prime}(x+y) & \geq 0, \\
-x \frac{v^{\prime \prime}}{v^{\prime}}(x+y) & \leq 1,
\end{aligned}
$$

and

$$
-x \frac{v^{\prime \prime \prime}}{v^{\prime \prime}}(x+y) \leq 1 .
$$

We have the following result.

Corollary 3 If the utility function of the representative investor exhibits risk aversion and prudence. Then, a positive (respectively negative) correlation of the exogenous risk and the endogenous risk causes a decrease (respectively an increase) in the price of the risky asset when relative risk aversion and relative prudence are less than one.

\subsection{Increasing the Correlation}

Epstein and Tanny (1980) show that an increase in the dependence parameter $k$ is disliked by any bivariate risk-averse representative investor. That is,

$$
k_{1} \leq k_{2} \Rightarrow\left(X_{2}, Y_{2}\right) \preccurlyeq 1,1-i c v\left(X_{1}, Y_{1}\right)
$$

where $k_{1}$ and $k_{2}$ are the respective dependence parameters of $\left(X_{1}, Y_{1}\right)$ and $\left(X_{2}, Y_{2}\right)$.

We now have the following result. 
Proposition 3 If $u \in \mathcal{U}_{2,1-i c v}$ with

$$
-x \frac{u^{(2,0)}}{u^{(1,0)}}(x, y) \leq 1 \text { and }-x \frac{u^{(2,1)}}{u^{(1,1)}}(x, y) \leq 1 \forall x, y
$$

then,

$$
k_{1} \leq k_{2} \Rightarrow P_{2}^{*} \leq P_{1}^{*}
$$

Proof $k_{1} \leq k_{2} \Rightarrow\left(X_{1}, Y_{1}\right) \preccurlyeq 1,1-i c v\left(X_{2}, Y_{2}\right)$, and applying Theorem 2 gives the result.

Increasing the dependence then decreases the price of the risky asset. In the univariate framework, by considering the utility function which accounts for additive risks $(u(x, y)=v(x+y))$, we have the following result.

Corollary 4 If the utility function of the representative investor exhibits risk aversion and prudence, an increase in the correlation of the exogenous risk and the endogenous risk causes a decrease in the price of the risky asset when relative risk aversion and relative prudence are less than one.

\section{Conclusion}

In this paper, we analyze the "monotonicity" of the asset prices by given conditions on preferences rather than restricting the change. We study the changes in the bivariate risk caused by the $(N, M)$-increasing concave order. We show that, due to the former changes, the price of the risky asset decreases when the utility function belongs to a particular class of functions and the coefficients of generalized relative risk aversion of order $(N, M)$ are less than $N$. Increasing the endogenous risk or the exogenous risk (marginal changes) via stochastic dominance induces a decrease in the market price when the coefficients of generalized relative risk aversion are less than their benchmark values. Positive correlation of the endogenous and the exogenous risks decreases the price of the risky asset whereas negative correlation increases it. Finally, increasing the correlation causes a decrease in the price of the risky asset. We also recover the results of the univariate framework.

Open Access This article is distributed under the terms of the Creative Commons Attribution 4.0 International License (http://creativecommons.org/licenses/by/4.0/), which permits unrestricted use, distribution, and reproduction in any medium, provided you give appropriate credit to the original author(s) and the source, provide a link to the Creative Commons license, and indicate if changes were made.

\section{References}

Cheng, H., Magill, M. J., \& Shafer, W. J. (1987). Some results in comparative statics under uncertainty. International Economic Review, 28, 493-507.

Choi, G., Kim, I., \& Snow, A. (2001). Comparative statics predictions for changes in uncertainty in the portfolio and savings problems. Bulletin of Economic Research, 53, 61-72. 
Denuit, M., Lefevre, C., \& Mesfioui, M. (1999). Stochastic ordering of convex-type for discrete bivariate risks. Scandinavian Actuarial Journal, 1, 32-51.

Dionne, G., Eeckhoudt, L., \& Gollier, C. (1995). Increase in risk and linear payoffs. International Economic Review, 34, 309-319.

Edwards, R. D. (2008). Health risk and portfolio choice. Journal of Business and Economic Statistics, 26, $472-485$.

Eeckhoudt, L., Etner, J., \& Schroyen, F. (2009). The values of relative risk aversion and prudence: A context-free interpretation. Mathematical Social Science, 58, 1-7.

Eeckhoudt, L., Liu, L., \& Meyer, J. (2016). Restricted increases in risk aversion and their application. Economic Theory, 64, 161-181.

Epstein, L. G., \& Tanny, S. M. (1980). Increasing generalized correlation: A definition and some economic consequence. Canadian Journal of Economy, 13, 16-34.

Fan, E., \& Zhao, R. (2009). Health status and portfolio choice: Causality or heterogeneity? Journal of Banking \& Finance, 33, 1079-1088.

Fishburn, P., \& Porter, B. (1976). Optimal portfolios with one safe and one risky asset: Effects of changes in rate of return and risk. Management Science, 22, 1064-1073.

Gollier, C. (1995). The comparative statics of changes in risk revisited. Journal of Economic Theory, 66, 522-535.

Gollier, C., \& Kimball, M. S. (2018). New methods in the classical economics of uncertainty: Comparing risks. The Geneva Risk and Insurance Review, 43, 5-23.

Gollier, C., \& Schlesinger, H. (2002). Changes in risk and asset prices. Journal of Monetary Economics, 49, 747-769.

Iwaki, H., \& Osaki, Y. (2017). Comparative statics and portfolio choices under the phantom decision model. Journal of Banking \& Finance, 84, 1-8.

Lucas, R. E. (1978). Asset prices in an exchange economy. Econometrica, 46, 1429-1446.

Meer, J., Miller, D., \& Rosen, H. S. (2003). Exploring the health-wealth nexus. Journal of Health Economics, 22, 713-730.

Ohnishi, M., \& Osaki, Y. (2007). The monotonicity of asset prices towards changes in risk. Economics, Management and Financial Markets, 2, 50-60.

Osaki, Y. (2005). Dependent background risk and asset prices. Economics Bulletin, 4(8), 1-8.

Palombo, M. G. (1999). Uncertain medical expenses and precautionary saving near the end of the life-cycle. Review of Economic Studies, 66, 395-421.

Pang, G., \& Warshawsky, M. (2010). Optimizing the equity-bond-annuity portfolio in retirement: the impact of uncertain health expenses. Insurance: Mathematics and Economics, 46, 198-209.

Richard, S. F. (1975). Multivariate risk aversion, utility independence and separable utility functions. Management Science, 22, 12-21.

Scarsini, M. (1985). Stochastic dominance with pair-wise risk aversion. Journal of Mathematical Economics, 14, 187-201. 